\title{
STUDIES ON THE EFFECT OF TOOL ROTATION IN MICRO- ULTRASONIC DRILLING OF HIGH ASPECT RATIO HOLES
}

\author{
Sandeep Kumar ${ }^{1}$, Akshay Dvivedi ${ }^{2}$ \\ ${ }^{1}$ Mechanical \& Industrial Engineering Department, IIT Roorkee, Uttarakhand, India 247667 \\ sandeepkumar71@gmail.com, \\ ${ }^{2}$ Mechanical \& Industrial Engineering Department, IIT Roorkee, Uttarakhand, India 247667 \\ akshaydvivedi@gmail.com
}

\begin{abstract}
Miniaturization is the need of hour as it saves the space, time, material, and energy as well. Therefore the demand of miniaturized features, components and products is rapidly increasing in industries. These micro-products includes the features such as microholes, and microchannels which are extensively used in microfluidics, Bio-MEMS, microelectronic chip (heat transfer) applications and Lab-on-a-chip. These micro-features are fabricated by conventional as well as non-conventional methods. Conventional methods include micro drilling whereas non-conventional techniques are Lithography, Chemical etching, Embossing, LIGA and Laser fabrication etc. Glass is commonly used material in these kind of application because it is chemically inert to most of the materials, have good optical properties, hydrophilic, non-porous suitable for prototyping and inexpensive as well. However being a fragile material, it is very difficult to machine by conventional method. Whereas LIGA, lithography and chemical etching are unable to generate high aspect ratio micro-features with good dimensional accuracy. Moreover these methods are time consuming and costly as well. Laser fabrication have the limitation to produce heat affected zone due to which workpiece properties change which further affect the useful life of product. Therefore there is a need to develop such process which can develop $3 D$ complex micro-features at faster rate without altering the properties of workpiece.
\end{abstract}

Keywords: Micro-USM, Tool rotation, Micro-hole, Borosilicate glass, Taper, Circularity $* * *$

\section{INTRODUCTION}

The demand of miniaturized product are increasing day by day because of their capability to save raw material, space, energy and time as well. The application area includes microfluidics, MEMS and lab-on-chip etc. (Lee and Lin 2004, Cheema et al. 2014). Glass and silicon are generally used in these type of applications. Micro-hole is one of the feature of miniaturized product. There are so many techniques such as conventional lithography based, and advanced machining process like micro-EDM, micro-USM, micro-ECDM, laser etc. are available to make micro-holes (Yu et al. 2004). Lithography techniques are limited to 2D surface only whereas micro-EDM, laser and micro-ECDM are use thermal energy to remove unwanted material and produce thermal damage on the workpiece surface which in turn affect the life of product (Singh et al. 2008). However to make an accurate micro-hole is still a challenge till date. Micro-USM is known to machine all types of brittle and hard material such as glass, ceramics silicon etc. (Sun et al. 1996, Mashuzawa and Tonshoff 1997). Another advantage of this process is, it does not change the properties workpiece material (Thoe et al. 1998). Material is removed in the form of micro-chips (debris) from the surface of workpiece due to the impact action of the abrasives followed by micro-chipping, cavitation erosion, and chemical action (Pandey and Shan 1980, Shaw 1956, Cook 1966, Miller 1957, Jain et al. 2011). This article reports on the development of a novel method to drill high aspect ratio micro-holes in borosilicate glass material. In the proposed study, rotation was provided to the vibrating tool to enhance the efficiency and quality of the generated feature. This process involves impacting abrasive particles which are suspended in a liquid slurry between rotating and vibrating tool and work piece. Further the effect of process parameters such as tool rotation speed and abrasive size was investigated on response characteristics. Circularity and taper were selected as process responses in this study.

\section{MATERIALS AND METHODS}

An in-house developed setup of rotary tool micro-USM was used in this study. Borosilicate glass material was selected as the workpiece material and silicon carbide was taken abrasive material. Tungsten carbide is a good candidate as the tool material for ultrasonic micromachining, thus it was selected a tool material in this study. Micro-holes were drilled at different parameter settings which are given below in Table 1. Tool rotation speed and slurry concentration were the variable process parameters whereas abrasive type, abrasive size, power rating and static load were the constant parameters in this study. Further, developed micro-holes were characterized using field emission electron microscope and optical microscope to check the quality characteristics such as circularity and taper. The rotary tool micro-USM setup and fabricated micro-hole are shown below in Figure 1.Circularity and taper were calculated by using the formulae given by equation $1 \& 2$. 


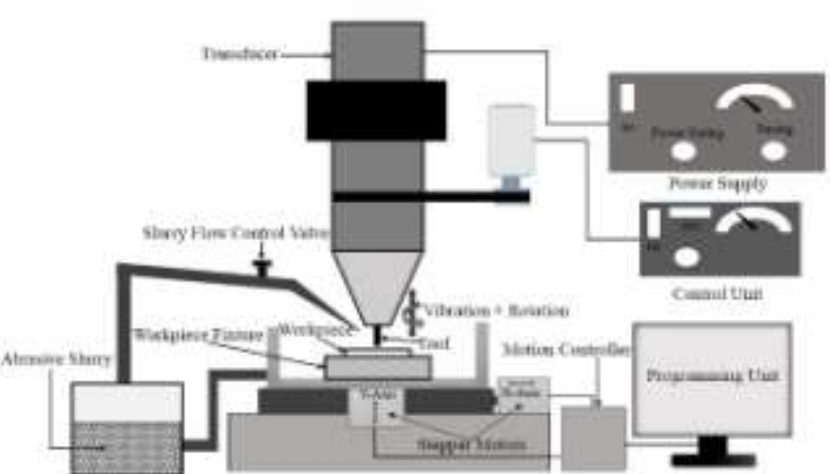

Figure 1: Schematic of rotary tool micro-USM (Kumar and Dvivedi, 2016)

Circularity $=D_{\min } / D_{\max }$

Where, $D_{\min } \& D_{\max }$ are minimum and maximum diameter of hole

Taper (degree $)=\frac{\left(D_{\text {avg }}\right)_{\text {entry }}-\left(D_{\text {avg }}\right)_{\text {exit }}}{\text { Thickness of workpiece }}$

Where, $\left(D_{a v g}\right)_{\text {antry }} \&\left(D_{a v g}\right)_{a x i t}$ are the average diameters of hole at entry and exit

Table 1. Process parameters setting

\begin{tabular}{|l|l|}
\hline Workpiece Material & Borosilicate Glass \\
\hline $\begin{array}{l}\text { Thickness of workpiece } \\
(\mathrm{mm})\end{array}$ & 1.35 \\
\hline Frequency $(\mathrm{kHz})$ & $25 \pm 1$ \\
\hline Tool Diameter $(\mu \mathrm{m})$ & 600 \\
\hline Tool Rotation Speed $(\mathrm{rpm})$ & $100,200,300,400$ \\
\hline Surry Concentration $(\%)$ & $\begin{array}{l}15,20,25 \text { (abrasive by } \\
\text { weight) }\end{array}$ \\
\hline $\begin{array}{l}\text { Abrasive Material \& mesh } \\
\text { size }\end{array}$ & SiC (\# 800) \\
\hline Power Rating $(\%)$ & 20 \\
\hline
\end{tabular}

\section{RESULT AND DISCUSSION}

Effect of tool rotation speed on circularity

Circularity is defined as the ratio of minimum diameter to maximum diameter at entry or exit. The ideal value of circularity is 1 (one). It was found from the experimentation that circularity improves with an increase in tool rotation speed (as shown in Figure 2). This can be attributed as tool rotation exerts centrifugal force in the slurry. The direction of the generated centrifugal force was outward from the center of the tool which forced the abrasives to move away easily. While moving outward, abrasive particles travel through the side gap between the tool and the wall of cavity. Some of the abrasives roll in the side gap between rotating tool and cavity wall. The rotary motion of tool also helped the abrasives to come out quickly from the side gap easily without damaging the periphery of hole. As a result of that the difference in between the maximum and minimum diameter of the generated micro-holes were very less which means circular holes were generated.

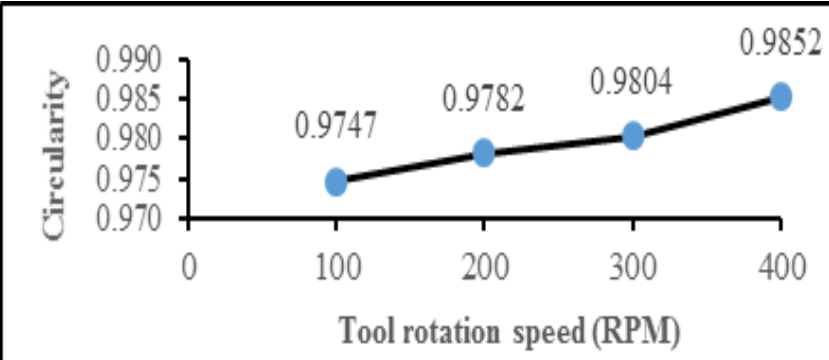

Figure 2: Effect of tool rotation speed on circularity

Effect of concentration on circularity

The effect of concentration on circularity is shown in Figure 3 . With an increase in concentration, circularity improved continuously. This can be explained as at low concentration, less number of abrasives were participated in the machining zone which resulted in non-uniform machining. Thus low value of circularity was obtained. On the other hand at higher value of concentration, more number of abrasives participated in the machining zone which resulted in uniform machining on workpiece surface which further resulted in the formation of circular holes.

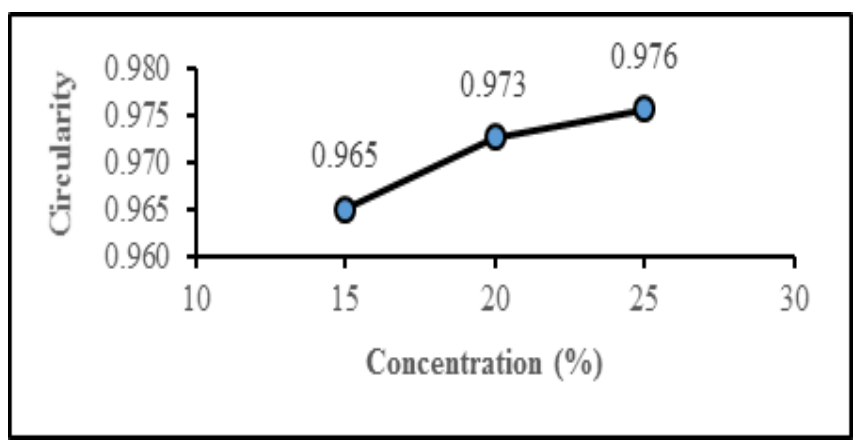

Figure 3: Effect of concentration on circularity

\section{Effect of tool rotation speed on taper}

The effect of tool rotation speed on taper is shown in Figure 4. It was found that with an increase in tool rotation speed, taper decreased. This can be attributed as tool rotation helped the abrasives to move easily from the machining zone without accumulating there. This resulted in smooth machining and very less tool wear. Which further resulted in very less difference in the entry and exit diameters of the generated hole. Thus low value of taper were obtained at increased rotation speed of the tool.

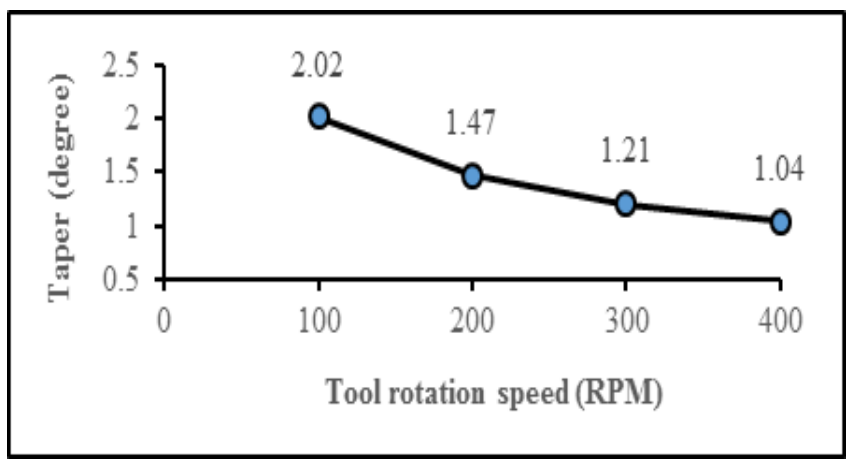

Figure 4: Effect of tool rotation speed on taper 


\section{Effect of concentration on taper}

The effect of concentration on taper is shown below in Figure 5. It can be seen that with an increase in concentration taper reduced marginally. This can be attributed as at higher value of concentration more abrasives were present in the gap between the rotating tool and workpiece which resulted in uniform layer of abrasive below the tool. This uniform layer resulted in good acoustic bond between the tool and workpiece which is desired for smooth machining. Due to this accurate holes of almost same entry and exit diameters (very low taper) were generated.

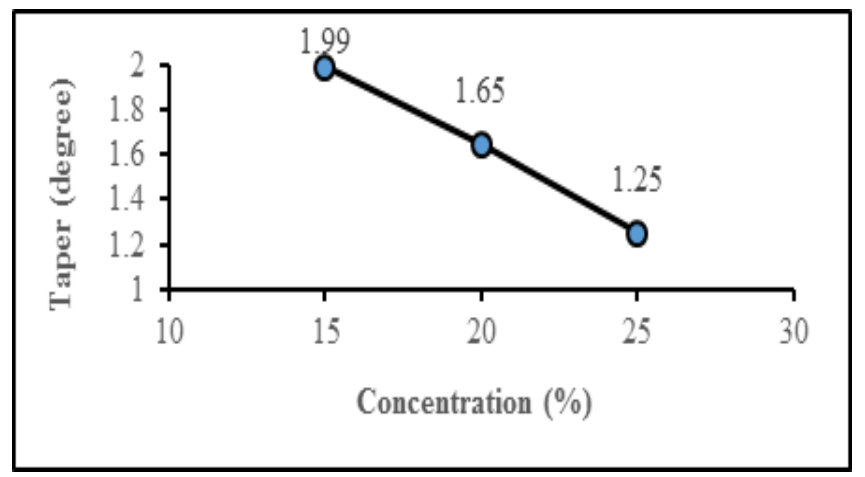

Figure 5: Effect of concentration on taper

Microscopic image of the fabricated micro-hole is shown in Figure 6. It can be seen from image that rotation of the tool resulted in less edge chipping near by the periphery of hole. This can be attributed as due to rotation of tool abrasives circulation became smooth and abrasive particles did not strike around the hole. In this proposed study micro-hole of up-to depth $2500 \mu \mathrm{m}$ and diameter $680 \mu \mathrm{m}$ was obtained.

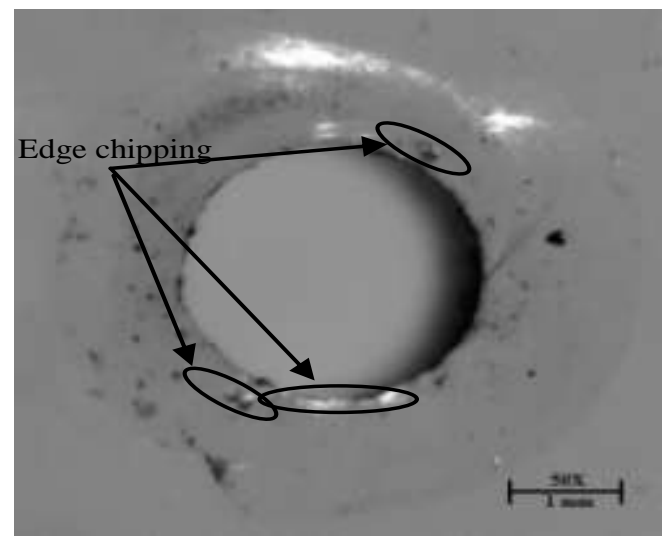

Figure 6: Microscopic image of hole

\section{CONCLUSION}

In this proposed study, an innovative method was developed for drilling of high aspect ratio micro-holes in glass workpiece. Rotation of tool resulted in significant improvement in the performance of the process. As a result of that geometrically accurate deep micro-holes were generated. During experimental investigation, the influence of process parameters were discussed. From the discussion of results it was found that circularity and taper of holes improved by increasing both the rotation speed of the tool and concentration of the abrasive slurry. Further, microscopic image revealed that rotary motion of the tool resulted in less edge chipping around the periphery of the hole.

\section{REFERENCES:}

[1]. Lee KB, Lin L (2004) Surface micromachined glass and polysilicon microchannels using mumps for biomems applications Sensors and Actuators A 111(1):44-50.

[2]. Cheema MS, Dvivedi A Sharma AK, Acharya S (2014) Experimental investigations in development of 3D microchannels through ultrasonic micromachining 9th International Workshop on Microfactories 92-95.

[3]. Yu ZY, Rajurkar KP, Tandon A (2004) Study of 3-D micro-ultrasonic machining Journal of Manufacturing Science and Engineering 126(4):727732.

[4]. Singh R, Alberts MJ, .Melkote SN (2008) Characterization and prediction of the heat-affected zone in a laser-assisted mechanical micromachining process International Journal of Machine Tools and Manufacture 48:994-1004.

[5]. Sun X, Masuzawa T, Fujino M (1996) Micro ultrasonic machining and its applications in MEMS Sensors and Actuators, A: Physical 57(2):159-164.

[6]. Masuzawa T, Tonshoff HK (1997) Threedimensional micro-machining by machine tools CIRP Annals - Manufacturing Technology 46(2):621-628.

[7]. Thoe TB, Aspinwall DK, Wise MLH (1998) Review on ultrasonic machining International Journal of Machine Tools and Manufacture 38(4):239-255.

[8]. Pandey PC, Shan HS (1980) Modern machining processes. Tata McGraw-Hill Education Pvt.. Ltd., ISBN: 13:9780070965539.

[9]. Shaw MC (1956) Ultrasonic grinding Microtechnic 10(6):257-265.

[10]. Cook NH (1966) Manufacturing analysis. AddisonWesley, New York 133-138.

[11]. Miller GE (1957) Special theory of ultrasonic machining Journal of Applied Physics 28(2):149 156.

[12]. Jain V, Sharma AK, Kumar P (2011) Recent developments and research issues in micro-ultrasonic machining ISRN Mechanical Engineering 1-11.

[13]. Kumar S, Dvivedi A (2016) Fabrication of 3D complex micro-features used in bio-medical applications Proceedings of the International Conference on Nanotechnology for Better Living 3(1):255. doi:10.3850/978-981-09-7519-7nbl16-rps255 . 